\title{
Enantioselective addition of diphenyl phosphonate to ketimines derived from isatins catalyzed by binaphthyl-modified organocatalysts
}

\author{
Hee Seung Jang, Yubin Kim and Dae Young Kim*
}

\section{Letter}

\section{Address:}

Department of Chemistry, Soonchunhyang University,

Soonchunhyang-Ro 22, Asan, Chungnam 31538, Korea

Email:

Dae Young Kim * - dyoung@sch.ac.kr

* Corresponding author

\section{Keywords:}

3-amino-3-phosphonyl-substituted oxindole; $\alpha$-aminophosphonates;

bifunctional organocatalyst; ketimines; organocatalysis; squaramide
Beilstein J. Org. Chem. 2016, 12, 1551-1556.

doi:10.3762/bjoc. 12.149

Received: 11 February 2016

Accepted: 06 July 2016

Published: 20 July 2016

This article is part of the Thematic Series "Bifunctional catalysis".

Guest Editor: D. J. Dixon

(ㄷ) 2016 Jang et al.; licensee Beilstein-Institut.

License and terms: see end of document.

\begin{abstract}
Chiral binaphthyl-modified squaramide-catalyzed enantioselective addition of diphenyl phosphonate to ketimines derived from isatins has been achieved. This method affords practical and efficient access to chiral 3-amino-3-phosphonyl-substituted oxindole derivatives in high yields with excellent enantioselectivities (up to $99 \%$ ee).
\end{abstract}

\section{Introduction}

$\alpha$-Aminophosphonate derivatives are important compounds as structural mimics of natural $\alpha$-amino acids [1-3]. Chiral $\alpha$-aminophosphonates have been shown a wide range of biological activities including antibacterial [4] and anticancer properties [5], enzyme inhibition [6], peptide mimetic function [7], and herbicidal properties [8]. Since the biological activity of $\alpha$-aminophosphonate derivatives is dependent upon the chirality of the $\alpha$-position to the phosphorus atom, asymmetric synthesis of $\alpha$-aminophosphonates has received considerable attention, and numerous catalytic enantioselective methods using chiral catalysts have been reported [9-13].

Oxindole and its derivatives can be exploited as important synthons to synthesize various alkaloid natural products and biologically active compounds [14-16]. In particular, 3,3-disubstituted oxindoles bearing a quaternary stereogenic center at the C3-position have been reported to be biologically active against a variety of targets [17-19]. Consequently, the asymmetric synthesis of 3,3-disubstituted oxindole derivatives has received significant research attention over the past few decades [20-22]. General approaches for the synthesis of chiral 3-substituted-3aminooxindole derivatives include the amination of various 3-monosubstituted oxindoles [23-27] and the nucleophilic addition to ketimines derived from isatin derivatives [28-35]. Recently, there were a few reports on the synthesis of chiral 3-amino-3-phosphonyl-substituted oxindole derivatives by the catalytic enantioselective hydrophosphonation of ketimines $[36,37]$. The previous synthetic procedures suffered from 
several drawbacks, such as a high catalyst loading, long reaction time, and low temperature required for good enantioselectivity. Thus, new approaches for the organocatalytic enantioselective addition of diphenyl phosphonate to isatin imines are highly desired.

In connection with our ongoing research program on the design and application in asymmetric catalysis of organocatalysts [3845], we have reported the catalytic asymmetric decarboxylative aldol addition reaction of isatins with benzoylacetic acids catalyzed by chiral binaphthyl-based squaramide [46]. Here we wish to report the enantioselective addition reaction of diphenyl phosphonate to ketimines derived from isatins catalyzed by binaphthyl-modified bifunctional organocatalysts (Figure 1).

\section{Results and Discussion}

To determine suitable reaction conditions for the organocatalytic enantioselective addition reaction of diphenyl phosphonate to ketimines derived from isatins, we initially investigated a reaction system with ketimine $\mathbf{1}$ a derived from $\mathrm{N}$-allylisatin and diphenyl phosphonate (2) with organocatalyst in the presence of $4 \AA$ molecular sieves. We first surveyed the effect of the structure of bifunctional organocatalysts I-VI (Figure 1) on enantioselectivity in ethyl acetate at room temperature (Table 1, entries 1-6). Catalyst III, which is a binaphthyl- modified squaramide bifunctional organocatalyst, was the best catalyst for this enantioselective addition reaction (90\% ee, Table 1, entry 3 ). In order to improve the selectivity, different solvents were tested in the presence of $10 \mathrm{~mol} \%$ of catalyst III together with ketimine 1a and diphenyl phosphonate (2). We obtained excellent results in ethyl acetate ( $85 \%$ yield, $90 \%$ ee, Table 1, entry 3), while a slight decrease in enationselectivities was observed when dichloromethane, chloroform, tetrahydrofuran, toluene, and methanol were used as the solvent (Table 1, entries 7-11). Under low catalyst loading of $2.5 \mathrm{~mol} \%$, this enantioselective addition reaction proceeded successfully to give 3a without compromising the reactivity and enantioselectivity (Table 1, entries 3 and 12-14). Finally, lowering the reaction temperature to $0{ }^{\circ} \mathrm{C}$ with catalyst III improved the enantioselectivity ( $93 \%$ ee, Table 1, entry 15 ). Performing the reaction without $4 \AA$ molecular sieves generated a lower yield (Table 1, entry 16).

With the optimized conditions in hand, we proceeded to investigate the scope of the enantioselective addition of diphenyl phosphonate (2) with various ketimines 1 in the presence of $2.5 \mathrm{~mol} \%$ of binaphthyl-modified squaramide-tertiary amine catalyst III in ethyl acetate at $0{ }^{\circ} \mathrm{C}$ (Table 2). The corresponding addition products $\mathbf{3 a}-\mathbf{l}$ were formed in high yields (74-94\%) with excellent enantioselectivities (up to 99\% ee). The reaction of diphenyl phosphonate (2) with $N$-allylated and<smiles>O=C(Nc1cc(C(F)(F)F)cc(C(F)(F)F)c1)NC1CCCCC1N1Cc2ccc3ccccc3c2-c2c(ccc3ccccc23)C1</smiles><smiles>O=C(Nc1cc(C(F)(F)F)cc(C(F)(F)F)c1)NC(c1ccccc1)[C@H](c1ccccc1)N1Cc2ccc3ccccc3c2-c2c(ccc3ccccc23)C1</smiles>

IV<smiles>FC(F)(F)c1cc(NC(=S)NC2CCCCC2N2Cc3ccc4ccccc4c3-c3c(ccc4ccccc34)C2)cc(C(F)(F)F)c1</smiles>

II<smiles>FC(F)(F)c1cc(NC(=S)NC(c2ccccc2)C(c2ccccc2)N2Cc3ccc4ccccc4c3-c3c(ccc4ccccc34)C2)cc(C(F)(F)F)c1</smiles>

V<smiles>O=c1c(Nc2cc(C(F)(F)F)cc(C(F)(F)F)c2)c(NC2CCCCC2N2Cc3ccc4ccccc4c3-c3c(ccc4ccccc34)C2)c1=O</smiles>

III<smiles>O=c1c(Nc2cc(C(F)(F)F)cc(C(F)(F)F)c2)c(NC(c2ccccc2)[C@H](c2ccccc2)N2Cc3ccc4ccccc4c3-c3c(ccc4ccccc34)C2)c1=O</smiles>

VI 
Table 1: Optimization of the reaction conditions. ${ }^{\text {a }}$

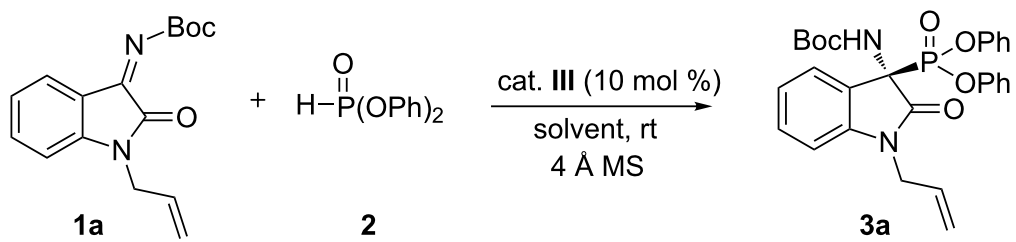

\begin{tabular}{|c|c|c|c|c|c|}
\hline entry & cat. & solvent & time $(\mathrm{h})$ & yield $^{\mathrm{b}}(\%)$ & $\mathrm{ee}^{\mathrm{c}}(\%)$ \\
\hline 1 & I & EtOAc & 9 & $3 a, 85$ & 73 \\
\hline 2 & II & EtOAc & 11 & $3 a, 94$ & 62 \\
\hline 3 & III & EtOAc & 9 & $3 a, 85$ & 90 \\
\hline 4 & IV & EtOAc & 12 & $3 a, 85$ & 54 \\
\hline 5 & V & EtOAc & 12 & $3 a, 85$ & 78 \\
\hline 6 & VI & EtOAc & 9 & $3 a, 95$ & 74 \\
\hline 7 & III & $\mathrm{CH}_{2} \mathrm{Cl}_{2}$ & 3 & $3 a, 92$ & 87 \\
\hline 8 & III & $\mathrm{CHCl}_{3}$ & 7 & $3 a, 82$ & 80 \\
\hline 9 & III & THF & 3 & $3 a, 88$ & 85 \\
\hline 10 & III & PhMe & 6 & $3 a, 75$ & 87 \\
\hline 11 & III & $\mathrm{MeOH}$ & 8 & $3 a, 54$ & 84 \\
\hline $12^{d}$ & III & EtOAc & 16 & $3 a, 82$ & 90 \\
\hline $13^{e}$ & III & EtOAc & 19 & $3 a, 80$ & 90 \\
\hline $14^{f}$ & III & EtOAc & 25 & $3 a, 76$ & 81 \\
\hline $15^{e, g}$ & III & EtOAc & 21 & $3 a, 80$ & 93 \\
\hline $16^{e, h}$ & III & EtOAc & 21 & $3 a, 58$ & 93 \\
\hline
\end{tabular}

aReaction conditions: ketimine (1a, $0.3 \mathrm{mmol})$, diphenyl phosphonate $(2,0.45 \mathrm{mmol})$, catalyst $(0.03 \mathrm{mmol})$, solvent $(3.0 \mathrm{~mL})$ in the presence of $150 \mathrm{mg}$ molecular sieves. ${ }^{\mathrm{b}}$ Isolated yield. ${ }^{C}$ Enantiopurity was determined by HPLC analysis using Chiralpak IB column. ${ }^{\mathrm{d}} 5 \mathrm{~mol} \%$ catalyst loading. ${ }^{\mathrm{e}} 2.5 \mathrm{~mol} \%$ catalyst loading. ${ }^{\mathrm{f}} 1.3 \mathrm{~mol} \%$ catalyst loading. ${ }^{\mathrm{g}}$ Reaction was performed at $0{ }^{\circ} \mathrm{C}$. ${ }^{\mathrm{h}}$ Reaction was performed without $4 \AA$ molecular sieves.

Table 2: Substrate scope. ${ }^{\text {a }}$

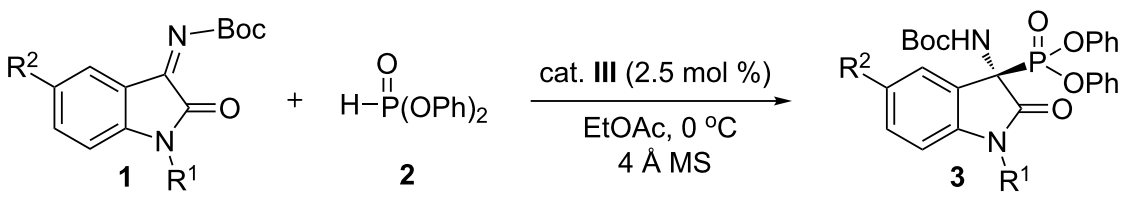

\begin{tabular}{|c|c|c|c|c|}
\hline entry & $1\left(R^{1}, R^{2}\right)$ & time $(\mathrm{h})$ & yield $(\%)^{\mathrm{b}}$ & ee $(\%)^{c}$ \\
\hline 1 & 1a $\left(\mathrm{R}^{1}=\mathrm{CH}_{2} \mathrm{CH}=\mathrm{CH}_{2}, \mathrm{R}^{2}=\mathrm{H}\right)$ & 21 & $3 a, 80$ & 93 \\
\hline 2 & $1 \mathbf{b}\left(\mathrm{R}^{1}=\mathrm{CH}_{2} \mathrm{CH}=\mathrm{CH}_{2}, \mathrm{R}^{2}=\mathrm{F}\right)$ & 15 & $3 b, 94$ & 94 \\
\hline 3 & 1c $\left(\mathrm{R}^{1}=\mathrm{CH}_{2} \mathrm{CH}=\mathrm{CH}_{2}, \mathrm{R}^{2}=\mathrm{Cl}\right)$ & 12 & $3 c, 90$ & 94 \\
\hline 4 & 1d $\left(\mathrm{R}^{1}=\mathrm{CH}_{2} \mathrm{CH}=\mathrm{CH}_{2}, \mathrm{R}^{2}=\mathrm{Br}\right)$ & 19 & $3 d, 84$ & 97 \\
\hline 5 & 1e $\left(\mathrm{R}^{1}=\mathrm{CH}_{2} \mathrm{C}\left(\mathrm{CH}_{3}\right)=\mathrm{CH}_{2}, \mathrm{R}^{2}=\mathrm{Cl}\right)$ & 48 & $3 e, 84$ & 99 \\
\hline 6 & 1f $\left(\mathrm{R}^{1}=\mathrm{CH}_{2} \mathrm{CH}=\mathrm{CHCH}_{3}, \mathrm{R}^{2}=\mathrm{Cl}\right)$ & 47 & $3 f, 70$ & 88 \\
\hline 7 & $\mathbf{1 g}\left(\mathrm{R}^{1}=\mathrm{CH}_{2} \mathrm{C}_{6} \mathrm{H}_{5}, \mathrm{R}^{2}=\mathrm{H}\right)$ & 21 & $3 g, 87$ & 99 \\
\hline 8 & 1h $\left(\mathrm{R}^{1}=\mathrm{CH}_{2} \mathrm{C}_{6} \mathrm{H}_{5}, \mathrm{R}^{2}=\mathrm{F}\right)$ & 20 & $3 h, 88$ & 99 \\
\hline 9 & $1 \mathbf{i}\left(\mathrm{R}^{1}=\mathrm{CH}_{2} \mathrm{C}_{6} \mathrm{H}_{5}, \mathrm{R}^{2}=\mathrm{Cl}\right)$ & 16 & $3 i, 78$ & 98 \\
\hline 10 & $\mathbf{1 j}\left(\mathrm{R}^{1}=\mathrm{CH}_{2} \mathrm{C}_{6} \mathrm{H}_{5}, \mathrm{R}^{2}=\mathrm{Br}\right)$ & 32 & $3 \mathbf{j}, 84$ & 99 \\
\hline 11 & $1 \mathbf{k}\left(\mathrm{R}^{1}=\mathrm{CH}_{2} \mathrm{C}_{6} \mathrm{H}_{5}, \mathrm{R}^{2}=\mathrm{OMe}\right)$ & 48 & $3 k, 79$ & 99 \\
\hline 12 & $1 \mathrm{I}\left(\mathrm{R}^{1}=\mathrm{H}, \mathrm{R}^{2}=\mathrm{Cl}\right)$ & 31 & 31,74 & 73 \\
\hline 13 & $1 \mathrm{~m}\left(\mathrm{R}^{1}=\mathrm{Boc}, \mathrm{R}^{2}=\mathrm{H}\right)$ & 48 & $3 \mathbf{m}, 45$ & 26 \\
\hline
\end{tabular}

aReaction conditions: ketimines $(1,0.3 \mathrm{mmol})$, diphenyl phosphonate $(2,0.45 \mathrm{mmol})$, catalyst (III, $7.5 \mu \mathrm{mol})$, EtOAc $(3.0 \mathrm{~mL})$ at $0{ }^{\circ} \mathrm{C}$ in the presence of $150 \mathrm{mg}$ molecular sieve. ${ }^{\mathrm{b}}$ Isolated yield. 'Enantiopurity was determined by HPLC analysis using Chiralpak IA (for $\mathbf{3 f}$ ), IB (for $\mathbf{3 a}$ ), IC (for $\mathbf{3 b}-\mathbf{e}, \mathbf{3 g}-\mathbf{j}$ ), and AD-H (for $\mathbf{3 k}, \mathbf{3 l}$ ) columns. 
5-halo- $N$-allylated isatin imines provided adducts $\mathbf{3 a - d}$ in good yields (80-94\%) with excellent enantioselectivities (93-97\% ee, Table 2, entry 1-4). The addition of diphenyl phosphonate (2) to 5-chloro- $N$-substituted isatin imines $\mathbf{1 e}$ and $\mathbf{1 f}$ provided 3-amino-3-phosphonyl-substituted oxindole derivatives $\mathbf{3 e}$ and 3f in high yields ( $84 \%$ and $70 \%$ ) with good enantioselectivities (99\% ee and $88 \%$ ee, Table 2, entries 5 and 6$). N$-Benzylisatin imine $\mathbf{1 g}$ and 5-halogen- $N$-benzylisatin imines $\mathbf{1 h}-\mathbf{j}$ reacted well with diphenyl phosphonate (2), giving 3-amino-3-phosphonyl-substituted oxindole derivatives $\mathbf{3 g}-\mathbf{j}$ in high yields (78-88\%) with excellent enantioselectivities (98-99\% ee) (Table 2, entries 7-10). Ketimine 1k containing an electron donating group gave the desired product $3 \mathbf{k}$ in high yield (79\%) with excellent enantioselectivity ( $99 \%$ ee, Table 2 , entry 11$)$. The nucleophilic addition of diphenyl phosphonate (2) to ketimine 21 derived from $N$-unprotected isatin was also studied. The adduct 31 was isolated in $74 \%$ yield with $73 \%$ ee (Table 2 , entry 12). Unfortunately, the reaction of diphenyl phosphonate (2) with $N$-Boc-ketimine $\mathbf{2} \mathbf{m}$ provided adduct $\mathbf{3 m}$ with low yield and enantioselectivity (Table 2 , entry 13). The absolute configuration of adducts 3 was determined to be $R$ by comparison of the specific rotations and HPLC properties with literature values [36.37].

The stereochemical outcome in the above addition reaction was rationalized by a proposed stereochemical model. We propose that ketimine $\mathbf{1}$ is activated by the squaramide moiety through hydrogen bonding, and diphenyl phosphonate (2) is activated by the basic nitrogen atom in the tertiary amine of catalyst III. Then, diphenyl phosphonate (2) attacks the re-face of the carbon in ketimine $\mathbf{1}$ as shown in Figure 2.

To further demonstrate the synthetic potential of this method, we performed the addition reaction at the gram scale. As shown in Scheme 1, when ketimine 1a was treated with diphenyl phosphonate (2) in the presence of $2.5 \mathrm{~mol} \%$ of catalyst III at $0{ }^{\circ} \mathrm{C}$, the desired product 3a was obtained in $81 \%$ yield and $93 \%$ ee (Scheme 1).

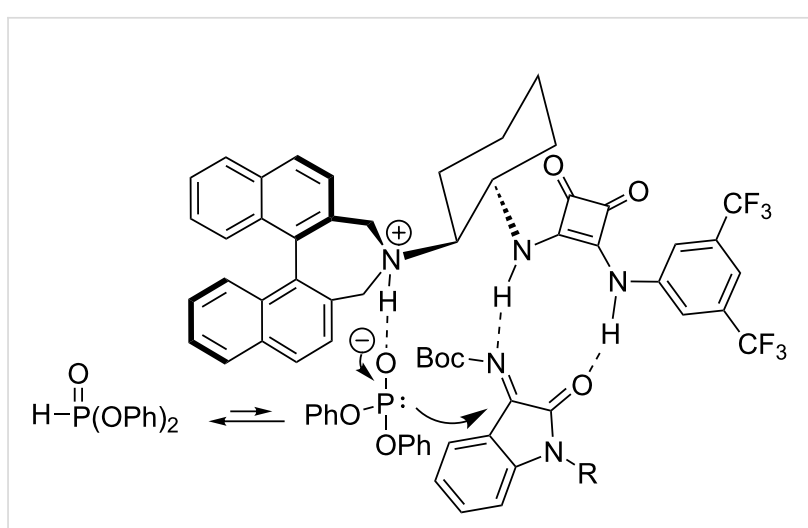

Figure 2: Proposed stereochemical model.

\section{Conclusion}

In conclusion, we have developed a practical and efficient catalytic enantioselective addition reaction of diphenyl phosphonate (2) with various ketimines 1 derived from isatins. This transformation is catalyzed by binaphthyl-modified squaramide catalyst III with low catalyst loading (2.5 mol \%). Chiral 3-amino-3-phosphonyl-substituted oxindole derivatives were obtained in high yields and excellent enantioselectivities were observed (up to $99 \%$ ee). This reaction affords valuable and easy access to chiral 3-amino-3-phosphonyl-substituted oxindole derivatives.

\section{Experimental}

General procedure for the enantioselective addition of diphenyl phosphonate (2) to ketimines derived from isatins 1: To a solution of ketimine $1(0.3 \mathrm{mmol})$, diphenyl phosphonate $(2,0.45 \mathrm{mmol})$, and $4 \AA$ molecular sieves $(150 \mathrm{mg})$ in ethyl acetate $(3 \mathrm{~mL})$, the catalyst (III, $7.5 \mu \mathrm{mol}$ ) was added at $0{ }^{\circ} \mathrm{C}$. The reaction mixture was stirred for 12-48 h. After completion of the reaction, the resulting solution was concentrated in vacuo and the obtained residue was purified by flash chromatography (EtOAc-hexane) to afford the corresponding adducts 3 .

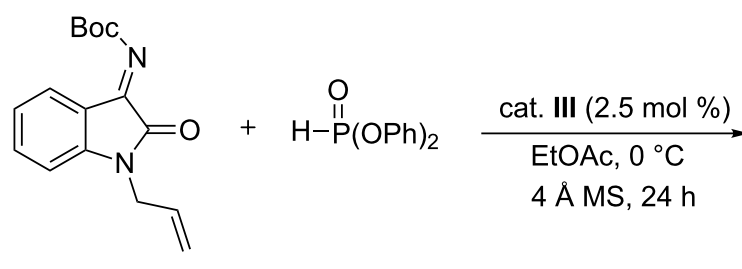

$1 \mathrm{a}(0.716 \mathrm{~g})$

$2(0.878 \mathrm{~g})$

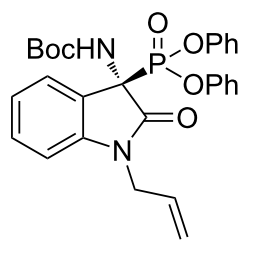

$3 a(1.051 \mathrm{~g})$

(81\% yield, $93 \%$ ee) 


\section{Supporting Information}

\section{Supporting Information File 1}

Experimental and analytical data.

[http://www.beilstein-journals.org/bjoc/content/

supplementary/1860-5397-12-149-S1.pdf]

\section{Acknowledgements}

This research was supported by the Soonchunhyang University Research Fund and Basic Science Research Program through the National Research Foundation of Korea (NRF) funded by the Ministry of Science, ICT and Future Planning (2014006224).

\section{References}

1. Berlicki, L.; Kafarski, P. Curr. Org. Chem. 2005, 9, 1829-1850. doi:10.2174/138527205774913088

2. Kafarski, P.; Lejczak, B. Curr. Med. Chem. 2001, 1, 301-312. doi:10.2174/1568011013354543

3. Moonen, K.; Laureyn, I.; Stevens, C. V. Chem. Rev. 2004, 104, 6177-6216. doi:10.1021/cr030451c

4. Xu, Y.; Yan, K.; Song, B.; Xu, G.; Yang, S.; Xue, W.; Hu, D.; Lu, P.; Ouyang, G.; Jin, L.; Chen, Z. Molecules 2006, 11, 666-676. doi:10.3390/11090666

5. Yao, G.-y.; Ye, M.-y.; Huang, R.-z.; Li, Y.-j.; Pan, Y.-m.; Xu, Q.; Liao, Z.-X.; Wang, H.-s. Bioorg. Med. Chem. Lett. 2014, 24, 501-507. doi:10.1016/j.bmcl.2013.12.030

6. Hu, D.-Y.; Wan, Q.-Q.; Yang, S.; Song, B.-A.; Bhadury, P. S.; Jin, L.-H.; Yan, K.; Liu, F.; Chen, Z.; Xue, W. J. Agric. Food Chem. 2008, 56, 998-1001. doi:10.1021/jf072394k

7. Hirschmann, R.; Smith, A. B., III; Taylor, C. M.; Benkovic, P. A.; Taylor, S. D.; Yager, K. M.; Sprengeler, P. A.; Benkovic, S. J. Science 1994, 265, 234-237. doi:10.1126/science.8023141

8. Barder, A. Aldrichimica Acta 1988, 21, 15.

9. Mucha, A.; Kafarski, P.; Berlicki, L. J. Med. Chem. 2011, 54, 5955-5980. doi:10.1021/jm200587f

10. Palacios, F.; Alonso, C.; de Los Santos, J. M. Chem. Rev. 2005, 105, 899-932. doi:10.1021/cr040672y

11. Gröger, H.; Hammer, B. Chem. - Eur. J. 2000, 6, 943-948. doi:10.1002/(SICI)1521-3765(20000317)6:6<943::AID-CHEM943>3.0. $\mathrm{CO} ; 2-4$ See for a review.

12. Ordonez, M.; Viveros-Ceballos, J. L.; Cativiela, C.; Azerpe, A. Curr. Org. Synth. 2012, 9, 310-341. doi:10.2174/157017912801270595

13. Vicario, J.; Ortiz, P.; Ezpeleta, J. M.; Palacios, F. J. Org. Chem. 2015, 80, 156-164. doi:10.1021/jo502233m

14. Marti, C.; Carreira, E. M. Eur. J. Org. Chem. 2003, 12, 2209-2219. doi:10.1002/ejoc. 200300050

15. Dounay, A. B.; Overman, L. E. Chem. Rev. 2003, 103, 2945-2964. doi:10.1021/cr020039h

16. Trost, B. M.; Brennan, M. K. Synthesis 2009, 3003-3025. doi:10.1055/s-0029-1216975

17. Liu, Y.; Han, S.-J.; Liu, W.-B.; Stoltz, B. M. Acc. Chem. Res. 2015, 48, 740-751. doi:10.1021/ar5004658
18. Galliford, C. V.; Scheidt, K. A. Angew. Chem., Int. Ed. 2007, 46, 8748-8758. doi:10.1002/anie.200701342

19. Zhou, F.; Liu, Y.-L.; Zhou, J. Adv. Synth. Catal. 2010, 352, 1381-1407. doi:10.1002/adsc.201000161

20. Kato, Y.; Furutachi, M.; Chen, Z.; Mitsunuma, H.; Matsunaga, S.; Shibasaki, M. J. Am. Chem. Soc. 2009, 131, 9168-9169. doi:10.1021/ja903566u

21. Tomita, D.; Yamatsugu, K.; Kanai, M.; Shibasaki, M. J. Am. Chem. Soc. 2009, 131, 6946-6948. doi:10.1021/ja901995a

22. Trost, B. M.; Czabaniuk, L. C. J. Am. Chem. Soc. 2010, 132, 15534-15536. doi:10.1021/ja1079755

23. Cheng, L.; Liu, L.; Wang, D.; Chen, Y.-J. Org. Lett. 2009, 11, 3874-3877. doi:10.1021/ol901405r

24. Qian, Z.-Q.; Zhou, F.; Du, T.-P.; Wang, B.-L.; Ding, M.; Zhao, X.-L.; Zhou, J. Chem. Commun. 2009, 6753-6755. doi:10.1039/B915257A

25. Bui, T.; Hernández-Torres, G.; Milite, C.; Barbas, C. F., III. Org. Lett. 2010, 12, 5696-5699. doi:10.1021/ol102493q

26. Mouri, S.; Chen, Z.; Mitsunuma, H.; Furutachi, M.; Matsunaga, S.; Shibasaki, M. J. Am. Chem. Soc. 2010, 132, 1255-1257. doi:10.1021/ja908906n

27. Shen, K.; Liu, X.; Wang, G.; Lin, L.; Feng, X. Angew. Chem., Int. Ed. 2011, 50, 4684-4688. doi:10.1002/anie.201100758

28. Montesinos-Magraner, M.; Vila, C.; Cantón, R.; Blay, G.; Fernández, I.; Muñoz, M. C.; Pedro, J. R. Angew. Chem., Int. Ed. 2015, 54, 6320-6324. doi:10.1002/anie.201501273

29. Bao, X.; Wang, B.; Cui, L.; Zhu, G.; He, Y.; Qu, J.; Song, Y. Org. Lett. 2015, 17, 5168-5171. doi:10.1021/acs.orglett.5b02470

30. Nakamura, S.; Takahashi, S. Org. Lett. 2015, 17, 2590-2593. doi:10.1021/acs.orglett.5b00805

31. Arai, T.; Tsuchiya, K.; Matsumura, E. Org. Lett. 2015, 17, 2416-2419. doi:10.1021/acs.orglett.5b00928

32. Takada, H.; Kumagai, N.; Shibasaki, M. Org. Lett. 2015, 17, 4762-4765. doi:10.1021/acs.orglett.5b02300

33. Engl, O. D.; Fritz, S. P.; Wennemers, H. Angew. Chem., Int. Ed. 2015, 54, 8193-8197. doi:10.1002/anie.201502976

34. Liu, T.; Liu, W.; Li, X.; Peng, F.; Shao, Z. J. Org. Chem. 2015, 80, 4950-4956. doi:10.1021/acs.joc.5b00302

35. Zhao, J.; Fang, B.; Luo, W.; Hao, X.; Liu, X.; Lin, L.; Feng, X. Angew. Chem., Int. Ed. 2015, 54, 241-244. doi:10.1002/anie. 201408730

36. George, J.; Sridhar, B.; Reddy, B. V. S. Org. Biomol. Chem. 2014, 12, 1595-1602. doi:10.1039/C3OB42026D

37. Kumar, A.; Sharma, V.; Kaur, J.; Kumar, V.; Mahajan, S.; Kumar, N.; Chimni, S. S. Tetrahedron 2014, 70, 7044-7049. doi:10.1016/j.tet.2014.06.013

38. Kang, Y. K.; Kim, S. M.; Kim, D. Y. J. Am. Chem. Soc. 2010, 132, 11847-11849. doi:10.1021/ja103786c

39. Kang, Y. K.; Lee, H. J.; Moon, H. W.; Kim, D. Y. RSC Adv. 2013, 3, 1332-1335. doi:10.1039/C2RA21945J

40. Kang, Y. K.; Kim, D. Y. Adv. Synth. Catal. 2013, 355, 3131-3136. doi:10.1002/adsc.201300398

41. Kang, Y. K.; Kim, D. Y. Chem. Commun. 2014, 50, 222-224. doi:10.1039/C3CC46710D

42. Suh, C. W.; Woo, S. B.; Kim, D. Y. Asian J. Org. Chem. 2014, 3, 399-402. doi:10.1002/ajoc.201400022

43. Suh, C. W.; Kim, D. Y. Org. Lett. 2014, 16, 5374-5377. doi:10.1021/ol502575f

44. Sung, H. J.; Mang, J. Y.; Kim, D. Y. J. Fluorine Chem. 2015, 178, 40-46. doi:10.1016/j.jfluchem.2015.04.021 
45. Kwon, S. J.; Kim, D. Y. Chem. Rec. 2016, 16, 1191-1203. doi:10.1002/tcr.201600003

46. Suh, C. W.; Chang, C. W.; Choi, K. W.; Lim, Y. J.; Kim, D. Y. Tetrahedron Lett. 2013, 54, 3651-3654.

doi:10.1016/j.tetlet.2013.04.132

\section{License and Terms}

This is an Open Access article under the terms of the Creative Commons Attribution License

(http://creativecommons.org/licenses/by/2.0), which permits unrestricted use, distribution, and reproduction in any medium, provided the original work is properly cited.

The license is subject to the Beilstein Journal of Organic Chemistry terms and conditions:

(http://www.beilstein-journals.org/bjoc)

The definitive version of this article is the electronic one which can be found at: doi:10.3762/bjoc. 12.149 